Eur Thyroid J 2020;9(suppl 1):1-2

DOI: $10.1159 / 000510702$

\title{
Twentieth EUGOGO Anniversary Symposium
}

The European Group on Graves' Orbitopathy (EUGOGO) organized a highly successful international 20th anniversary meeting on November 7-9, 2019, in the beautiful city of Pisa, Italy. More than 200 experts and key opinion leaders in the field of Graves' orbitopathy (GO) or thyroid eye disease and thyroid autoimmunity gathered in Tuscany to discuss the latest findings in experimental research, animal models, pathogenesis, genetics, and immunology of this complex orbital disease. Further, developments of diagnostic procedures, for example, imaging and serology, as well as surgical approaches were extensively presented. Basic scientists, immunologists, endocrinologists, thyroidologists, specialists in nuclear medicine, ophthalmologists, and orbital and ENT surgeons actively participated. The scientific program was an elegant mixture of reviews and updates delivered by acknowledged international experts and sessions focused on recent advances in GO. These oral sessions offered young investigators the opportunity to present their novel data to the plenary audience as no parallel activities were foreseen. The only exception was an excellently organized, well-attended lively session for patients with GO (mostly from Italy), which took place on Saturday morning, November 9, 2020, and which was moderated by the local organizers and other Italian colleagues.

The EUGOGO event was so successful due to the following reasons:

EUGOGO has an established, well-organized infrastructure and a closely cooperating executive committee (EC). This effective interaction between the principal officers and members of the EUGOGO EC led to the draft of an attractive, multidisciplinary, up-to-date scientific program.

The local organizing committee (LOC) chaired by Claudio Marcocci, Michele Marino, member of the EU-
GOGO EC, and their coworkers did an outstanding job pertaining to selecting the congress venue, the effective congress agency (Mrs. Roberta Bertuccelli and Mrs. Cristina de Rosa, "Asti Incentives \& Congressi"), and the various locations for the attractive and pleasant social activities. Everything went smoothly due to the well-orchestrated and excellent management of the symposium.

The above points may explain why all invited key opinion leaders and international experts from Asia, Europe, and the Americas spontaneously accepted the EUGOGO invitation, which allowed for the gathering of a highly qualified expert faculty.

In addition, the EUGOGO EC and the LOC were most pleased to welcome more than 65 abstract submissions from more than 20 countries dealing with various aspects of GO. Both the oral presentations and the poster sessions engendered lively discussions.

Of course, such a well-attended international meeting with a large expert faculty requires a solid budget. EUGOGO, the principal officers, and the LOC are most grateful to Horizon Therapeutics, USA for generously sponsoring this event. The appreciated logistical support greatly facilitated the timely planning and selection of sites for the congress venue and social program. Fortunately and additionally, several Italian sponsors kindly offered financial assistance.

As several novel and challenging aspects pertaining to the pathogenesis and management of GO were introduced to the audience, the EUGOGO EC and the LOC decided to publish within a special issue of the European Thyroid Journal (ETJ), official journal of the European Thyroid Association, a selection of up-to-date review and research articles written by internationally acknowledged experts. These chapters deal with (a) the TSH and IGF-1 receptors as key autoantigens in GO and their cross talk 
(Gershengorn, Neumann et al., USA), (b) novel small molecules inhibiting the TSH receptor through binding to the transmembrane domain (Krause et al., Germany), (c) the role of the microbiome in GO (Ludgate et al., UK), (d) oxidative mechanisms in GO (Marino, Marcocci et al., Italy), (e) a long-term animal model for Graves' disease and associated GO (Ungerer et al., Germany), (f) new recommendations for the objective assessment of clinical activity and severity of GO (Bartalena \& Wiersinga, Italy and the Netherlands), (g) novel therapeutic approaches for Graves' hyperthyroidism and for GO (Lee et al., Hong Kong), and finally (h) a review on the anti-IGF-1R inhibiting monoclonal antibody, teprotumumab (Smith, USA).

It has been a great pleasure and an honor for Claudio Marcocci and myself to edit this ETJ special issue celebrating the 20th anniversary of EUGOGO. We are most grateful to (a) all expert authors for timely delivering ex- cellent and up-to-date manuscripts; (b) to Professor Simon Pearce, editor-in-chief of the ETJ, for the smooth and most pleasant cooperation; (c) to Patrick Näf, production editor at Karger Publishers, for an excellent task and for his appreciated reliability; (d) to Dr. sc. Jessada Mahatthananchai, Karger publication manager, for the successful contract agreement with the sponsor; and (e) to Jeffrey Sherman, MD, FACP, executive vice-president and chief medical officer, Horizon Therapeutics, for his much-appreciated personal and logistical support of the scientific event as well as of this special issue.

Enjoy the reading!

With all best wishes, George J Kahaly, Treasurer of EUGOGO, Mainz, Germany

Claudio Marcocci, Chairman of EUGOGO, Pisa, Italy 